\title{
Long-term in situ permafrost thaw effects on bacterial communities and potential aerobic respiration
}

\author{
Sylvain Monteux $\mathbb{1}^{1} \cdot$ James T. Weedon $\mathbb{1}^{2,3} \cdot$ Gesche Blume-Werry ${ }^{1} \cdot$ Konstantin Gavazov ${ }^{1,4}$. \\ Vincent E. J. Jassey $\mathbb{D}^{5} \cdot$ Margareta Johansson $^{6} \cdot{\text { Frida Keuper } \mathbb{D}^{7} \cdot \text { Carolina Olid }^{1} \cdot \text { Ellen Dorrepaal }}^{1}$
}

Received: 21 September 2017 / Revised: 15 March 2018 / Accepted: 28 March 2018 / Published online: 6 June 2018

(c) The Author(s) 2018. This article is published with open access

\begin{abstract}
The decomposition of large stocks of soil organic carbon in thawing permafrost might depend on more than climate change-induced temperature increases: indirect effects of thawing via altered bacterial community structure (BCS) or rooting patterns are largely unexplored. We used a 10-year in situ permafrost thaw experiment and aerobic incubations to investigate alterations in BCS and potential respiration at different depths, and the extent to which they are related with each other and with root density. Active layer and permafrost BCS strongly differed, and the BCS in formerly frozen soils (below the natural thawfront) converged under induced deep thaw to strongly resemble the active layer BCS, possibly as a result of colonization by overlying microorganisms. Overall, respiration rates decreased with depth and soils showed lower potential respiration when subjected to deeper thaw, which we attributed to gradual labile carbon pool depletion. Despite deeper rooting under induced deep thaw, root density measurements did not improve soil chemistry-based models of potential respiration. However, BCS explained an additional unique portion of variation in respiration, particularly when accounting for differences in organic matter content. Our results suggest that by measuring bacterial community composition, we can improve both our understanding and the modeling of the permafrost carbon feedback.
\end{abstract}

Electronic supplementary material The online version of this article (https://doi.org/10.1038/s41396-018-0176-z) contains supplementary material, which is available to authorized users.

Sylvain Monteux

monteux@protonmail.com

1 Climate Impacts Research Centre (CIRC), Department of Ecology and Environmental Sciences, Umeå Universitet, 98107 Abisko, Sweden

2 Systems Ecology, Department of Ecological Sciences, Vrije Universiteit Amsterdam, 1081 HV Amsterdam, The Netherlands

3 PLECO, Department of Biology, University of Antwerp, 2610 Wilrijk, Belgium

4 Federal Institute for Forest, Snow and Landscape Research WSL, CH-1015 Lausanne, Switzerland

5 Functional Ecology and Environment Laboratory (ECOLAB), Department of Biology and Geosciences, UMR 6245 Université Toulouse III Paul Sabatier, 31062 Toulouse cedex 09, France

6 Department of Physical Geography and Ecosystem Science, Lund Universitet, 22362 Lund, Sweden

7 INRA, AgroImpact UR1158, Site Laon, 02000 Barenton Bugny, France

\section{Introduction}

Northern hemisphere permafrost soils and their overlying active (seasonally thawing) layer store $1035-1580 \mathrm{Pg}$ of organic carbon, about $28 \%$ of which is found in peat deposits [1, 2]. This carbon stock is protected from decomposition due to limited microbial activity in frozen soils [3, 4]. Climate change-induced thawing of permafrost soils stimulates decomposition of this carbon, potentially causing a positive feedback to warming $[2,5]$. However, it remains uncertain how permafrost thaw will affect soil organic matter (SOM) decomposition in the long term, and how this relates to changes in other potential drivers of SOM decomposition, such as bacterial communities and plant root distribution.

While higher temperatures increase soil respiration in laboratory incubations [6], field studies have shown contrasting results, with increased but also reduced responses of soil respiration to experimental warming and permafrost thaw in the longer term (e.g. [7, 8]). Long-term decreases in soil respiration have variously been attributed to depletion of labile carbon substrates or microbial acclimation [9]. 
Decomposition in natural thawing permafrost soil may therefore be controlled not only by increases in temperature but also by various associated changes in the ecosystem. Permafrost thaw can, for example, alter other soil abiotic conditions, such as nutrient availability [10], which can in turn affect decomposition rates [11]. In the longer term, active layer-deepening can favor deeper-rooting plant species [12, 13], which might increase SOM decomposition through rhizodeposition of fresh carbon (priming effects [14-16]). Deep roots in permafrost peatlands might stimulate SOM decomposition through increased oxygen availability if they harbor aerenchymae [17], as anoxia partly inhibits SOM decomposition in peatlands [18]. Moreover, changes in abiotic soil properties or rooting patterns may affect decomposition indirectly through altering microbial community structure $[19,20]$. In the long term, SOM decomposition is therefore likely altered by permafrost thaw due to changes in temperature, root density, abiotic soil conditions, or microbial community structure, but, so far, the extent and underlying ecological interactions remain unclear.

Microorganisms are important decomposers, especially in deeper soil layers, which larger soil fauna cannot access $[21,22]$. Nonetheless, the importance of microbial community structure for decomposition rates is controversial [23]. On the one hand, soil microbial communities are often considered to exhibit high functional redundancy with regard to decomposition, making their composition unimportant for determining process rates [24]. On the other hand, microbial community structure may affect decomposition in some systems, e.g., when microbial diversity is very low [25]. Deeper, permanently frozen soil layers exhibit lower microbial biomass, diversity, and functional potential than the active layer, due to long-term adaptation to frozen conditions [26, 27]. Bacteria have higher abundance than archaea and fungi in permafrost subsoil systems [26, 28, 29] and are important contributors to respiration of peatland soils, especially at high water content [30, 31]. Bacterial community structure (BCS; sensu taxonomic composition) could therefore be an important determinant of decomposition in thawing permafrost soils, but so far, this link has not been directly evaluated.

BCS may be affected by warming-induced changes to plant community composition and rooting behavior [12, 32, $33]$, through altered rhizosphere extent and properties [20, 34]. These effects will be strongest in the newly thawed permafrost at the base of the active layer, where no roots were previously present. Thawing permafrost soils will also experience physico-chemical changes: phase transition in the newly thawed permafrost will remove the long-term freezing constraints (e.g., low water availability and energy input) with potentially large effects on bacterial communities $[28,35]$. Climate change and permafrost thaw are thus likely to modify bacterial communities through alterations in soil abiotic factors and plant root distribution, with the strongest effects in the newly thawed soil layers.

Common approaches to study the microbial ecology of thawing permafrost are natural thaw gradients (e.g. [26]) and laboratory incubations (e.g. [28]). Natural thaw gradients provide realistic proxies for future effects of climate change, but the history of deep-thaw sites may not resemble current shallow-thaw sites. BCS can vary strongly with depth within a soil profile [36] and the thawing of permafrost induces varying soil subsidence and compaction depending on ice content [37]. Therefore, ensuring that the communities compared along a presumed thaw gradient were not different before permafrost thaw occurred can be challenging. Such potential artifacts are avoided by repeated sampling from thawing and incubating permafrost samples but in vitro approaches ignore indirect effects of thawing, such as through soil fauna and root colonization. In contrast, inducing permafrost thaw in the field, e.g., by experimentally increasing winter insulation with snow fences to simulate winter climate change $[38,39]$, allows for the detection of these longer-term indirect effects of thawing. Such an experimental setup also allows for more precision in sampling soil from a given depth as subsidence can be monitored. Furthermore, by careful selection of plots and randomization of treatments initially identical microbial communities are ensured, precluding the potential confounding effects inherent to spatial gradients.

We investigated how the combined direct and indirect effects of long-term in situ permafrost thaw through experimental winter-warming modifies the BCS and potential soil respiration across the active layer and upper permafrost soil, and studied whether and how these responses were linked. We hypothesized that:

(a) Decadal in situ winter-warming and associated permafrost thaw will modify BCS across active layer and permanently frozen soil, most strongly at the depth of the newly thawed and recently root-colonized soil.

(b) Potential respiration will decrease with decadal winter-warming in the active layer and increase in the newly thawed permafrost soil. This is because of long-term depletion of labile carbon substrates after a decade of higher temperatures in the active layer. In contrast, the intermediate layer has only thawed for a short duration at the end of each growing season, so we expect increased respiration as a result of changes in BCS (e.g., by shifts toward communities with higher functional diversity).

(c) Including BCS-i.e., phylogeny-informed taxonomic composition-and plant root density will improve soil chemistry-based predictions of potential respiration across active layer and thawing permafrost soil. 


\section{Materials and methods}

\section{Deep-thaw experiment}

The study site is located in the Storflaket palsa peatland close to Abisko, Northern Sweden (68.346N, 18.971E), of which active layer thickness (ALT) was $69.3 \pm 3.7 \mathrm{~cm}$ in 2015 , and water table depth was $30.2 \pm 2.8 \mathrm{~cm}$ in October 2013 (mean \pm SE). The peatland is dominated by peat moss (Sphagnum spp.), Eriophorum vaginatum L., Vaccinium vitis-idaea L., Andromeda polifolia L., Betula nana L., Empetrum nigrum L. and Rubus chamaemorus L. Since 2005, $10 \mathrm{~m}$ long and $1 \mathrm{~m}$ high snow fences were erected each winter in six randomly chosen plots out of $12(10 \times 10$ $\mathrm{m})$, increasing mean winter snow accumulation 2.6-fold (16-24 cm), winter soil temperature (at $15 \mathrm{~cm}$ ) by $1.5^{\circ} \mathrm{C}$, and ALT by $29 \mathrm{~cm}$ (in 2015, data not shown), and are further referred to as "deep thaw". The remaining six plots, with ambient snow accumulation $(6-9 \mathrm{~cm})$ served as controls. Effects include an increase in E. vaginatum growth and cover and in soil subsidence (average $24 \mathrm{~cm}$ in 2012 compared to 2005 , vs $5 \mathrm{~cm}$ in control plots) causing seasonal ponds in some deep-thaw plots. Methane emissions were nonsignificant within or outside of the deep-thaw plots ( $0.09 \pm 0.08$ and $0.1 \pm 0.18 \mathrm{mg} \mathrm{CH}_{4} / \mathrm{m} / \mathrm{h}$, respectively [40]), therefore we did not investigate methane dynamics. More details are found in ref. [41].

\section{Sampling design and sampling for BCS, plants, and abiotic analyses}

We compared subsoil carbon and microbial dynamics around the thawfront at three depths: active layer (above the thawfront but below the water table in both deep-thaw and control plots, ca. $55 \mathrm{~cm}$ ); intermediate layer (frozen in control plots, seasonally thawed in deep-thaw plots, ca. 70 $\mathrm{cm}$ ); and permafrost layer (perennially frozen in both deepthaw and control plots, at least $10 \mathrm{~cm}$ below the thawfront, $100-125 \mathrm{~cm})$.

Two soil cores per plot were collected in September 2015, around the time of maximum ALT [41], using a peat-corer for thawed soil $(11 \times 11.4 \mathrm{~cm}$, Eijkelkamp, The Netherlands), and a custom-made gas-powered fluid-less concrete drill for permafrost soil $(10.2 \mathrm{~cm}$ diameter). As the ALT varies with distance to the fences within deepthaw plots, we selected spots with $\mathrm{ALT}>80 \mathrm{~cm}$ for coring, but we did not use ALT criteria for the cores in control plots (mean \pm SE for control and deep-thaw cores were $60.1 \pm 2.4$ and $90.2 \pm 6.4 \mathrm{~cm}$, respectively; $P<0.001$, $n=12$ ). Frozen soil cores were rinsed with sterile deionized water to limit drilling contamination between soil layers, wrapped in plastic foil and stored in a cooler box for up to $3 \mathrm{~h}$.
One set of cores was thawed at $2{ }^{\circ} \mathrm{C}$ after which $2 \mathrm{~g}$ soil was sampled for DNA extraction with ethanol-cleaned forceps, at least $3 \mathrm{~cm}$ from the core surface to minimize contamination, from the three aforementioned depths.

Three 30-cm segments were taken encompassing these sampling depths (i.e., 30-60, 60-90, >90 cm), and about 1 $\mathrm{L}$ (estimated by water displacement) of each core segment was used for manually collecting coarse roots $(>0.5 \mathrm{~mm})$, which were dried at $60{ }^{\circ} \mathrm{C}$ and weighed to calculate density of roots in the soil ( $\mathrm{g}$ dry weight roots/L soil). Due to sampling errors, one replicate of the intermediate layer in deep-thaw plots was absent from this set of cores, and therefore excluded from analyses including BCS and root density.

The second set of cores was stored at $-20^{\circ} \mathrm{C}$ until April 2016, then thawed at $4{ }^{\circ} \mathrm{C}$ and subsamples were taken for soil chemistry analyses and aerobic incubations. These subsamples were homogenized by sieving through an ethanol-cleaned $2 \mathrm{~mm}$ sieve. Homogenized soil aliquots were used to measure potential aerobic respiration (see below), as an indicator of the vulnerability of soil carbon. Further, we used $\sim 9 \mathrm{~g}$ subsamples to determine gravimetric water content $\left(60^{\circ} \mathrm{C}, 24 \mathrm{~h}\right)$ and organic matter content (OMC, $475^{\circ} \mathrm{C}, 2 \mathrm{~h}$ [42]). Carbon content (g C/g dry soil) was analyzed using a NCS 2500 elemental analyzer (CE Instruments, Milan, Italy). Another $3 \mathrm{~g}$ (fresh weight) were shaken in $40 \mathrm{~mL}$ of sterile deionized water for $2 \mathrm{~h}$ and filtered. $\mathrm{pH}$ of the filtrate was measured on a MP220 pH meter (Mettler-Toledo, Greifensee, Switzerland) and $\mathrm{NH}_{4}{ }^{+}$and $\left(\mathrm{NO}_{3}{ }^{-}+\mathrm{NO}_{2}{ }^{-}\right)$were quantified on a FIAstar 5000 Autoanalyzer (FOSS Analytics, Hilleroed, Denmark). Nitrate and nitrite concentrations were below detection limit (ca. $12.2 \mu \mathrm{g} \mathrm{NO}_{3}{ }^{-}-\mathrm{N} / \mathrm{g}$ dry soil) in most samples and are therefore not discussed further.

\section{Soil incubations}

For each of the six replicates at three sampling depths in the two treatments, two aliquots of $20 \mathrm{~g}$ (fresh weight) homogenized soil were put into $250 \mathrm{~mL}$ glass jars closed with rubber septa and incubated in dark culture chambers at either 11 or $21^{\circ} \mathrm{C}$. Headspace air $(10 \mathrm{~mL})$ was sampled with an airtight glass syringe and $\mathrm{CO}_{2}$ concentrations were measured with an infrared gas analyzer (EGM-4, PP Systems, Amesbury, Massachusetts), using the instrument's internal calibration and static sampling mode, at 1-5 days' intervals for 1 month. When one sample reached $\geq 10000$ ppm, all jars were flushed with 400 ppm $\mathrm{CO}_{2}$ synthetic air, long enough to lower the headspace $\mathrm{CO}_{2}$ concentration to $400 \mathrm{ppm}$. One month after homogenization, respiration rates stabilized and four more measurements were taken, each time starting at $400 \mathrm{ppm}$ (achieved by flushing as described above) and continuing for 1-2 days. These four 
measurements were used to calculate $\mathrm{CO}_{2}$ production rates $(\tau)$ as follows:

$$
\tau_{(1-2)}=\frac{n_{2}-n_{1}}{\left(\Delta_{t}\right)_{1-2}} \text { with } n_{i}=\left[\mathrm{CO}_{2}\right]_{i} \times\left(P_{i} V / R T\right)
$$

where $\left(\Delta_{t}\right)_{1-2}$ is the time interval between flushing $\left(_{1}\right)$ and measurement $(2), P i$ is atmospheric pressure at flushing or measurement time (data from Abisko Naturvetenskapliga Station), $V$ the headspace volume, $R$ the ideal gas constant, and $T$ the incubation temperature. The four rates were then averaged to estimate the potential aerobic respiration rates (hereafter respiration).

Temperature sensitivity of respiration $\left(Q_{10}\right.$ [43]) was calculated for each replicate soil sample based on these averaged values at 11 and $21^{\circ} \mathrm{C}$. Potential respiration rates were expressed either per soil dry weight (bulk respiration) or per organic matter dry weight (intrinsic respiration).

\section{Bacterial communities}

DNA was extracted using a PowerSoil DNA Extraction Kit (Qiagen, Venlo, The Netherlands), following the manufacturer's instructions. DNA was quantified using QuantIT dsDNA assay (Thermo Scientific, Waltham, Massachusetts, SI Fig S1). The DNA extracts were diluted to $5 \mathrm{ng} / \mu \mathrm{L}$ with nuclease-free water. Samples with concentrations $<10 \mathrm{ng} /$ $\mu \mathrm{L}$ were diluted $1: 1$.

The V3 region of the $16 \mathrm{~S}$ ribosomal RNA gene was targeted in PCR amplification (341F; 518R, SI Table S1) to characterize BCS [44], and quality of amplification was visually checked by gel electrophoresis. Amplicons were cleaned and normalized using SequalPrep Normalization Plate Kit (Thermo Scientific), then pooled, and further purified using a QIAquick Gel Extraction Kit (Qiagen, Venlo, The Netherlands). The resulting pooled library was sequenced on an Illumina MiSeq with V2 chemistry and $2 \times 150$ bp paired-end reads (BioSample accession numbers SAMN07445364-SAMN07445398 and SAMN07445422).

All bioinformatics scripts are available as a Jupyter notebook at https://bitbucket.org/smonteux/monteux_deep_ thaw. Merging, quality filtering, and $97 \%$ de novo operational taxonomic unit (OTU) clustering were performed with VSEARCH v1.10.2 [45], chimera removal with UCHIME, and the GOLD database [46, 47]. OTUs that were abundant in the technical control sample (>5\%) were removed, alike in ref. [48]. PyNAST, FastTree, the RDPnaive Bayesian classifier, and Greengenes 13.8 database were used in QIIME v1.9.1 [49-52] to obtain OTUs taxonomy, filtering out OTUs present in $<10 \%$ of the samples to exclude highly variable OTUs that may inflate the number of differentially abundant OTUs (adapted from ref. [53]). Weighted UniFrac distances [54] were computed on an abundance table averaged from 100 rarefactions at
899 sequences depth. Differential abundance of OTUs between treatments for each soil layer was assessed using DESeq 2 negative-binomial Wald test on non-rarefied reads $[55,56]$, and indications of the ecology of the most abundant OTUs ( $>0.5 \%$ of rarefied reads) affected by the deepthaw manipulation were inferred based on their taxonomy and the ecology of their closest relatives (BLAST [57], SI Table S2 [58-72]).

\section{Statistical analyses}

All statistical analyses were carried out using R v3.4.1 [73], and the script used to produce figures and tables is available at https://bitbucket.org/smonteux/monteux_deep_thaw. Effects of the deep-thaw treatment and soil depth on potential respiration at $11^{\circ} \mathrm{C}, Q_{10}$, and soil abiotic variables (OMC, $\mathrm{pH}, \mathrm{NH}_{4}{ }^{+}$, and soil moisture) were assessed using analysis of variance with linear mixed-effects models (nlme package [74]), using treatment and depth as fixed factors, and core as a random factor, followed by pairwise contrasts when appropriate (lsmeans package [75]). $\mathrm{NH}_{4}^{+}$and both bulk and intrinsic potential respiration were log-transformed, and OMC was square-root-transformed to improve normality of residuals.

The effects of depth and treatment on BCS were assessed by testing for deviation of each OTU against a negativebinomial distribution with generalized linear models (manyglm(), mvabund package [76]), which confounds location and dispersion effects less than distance-based approaches (e.g., PERMANOVA [77]). A principal coordinates analysis (PCoA) ordination of weighted UniFrac distances was computed from the averaged abundance table and associations with root density and soil variables were tested using envfit() (vegan package [78]).

To explore relationships between potential respiration on one hand, and soil chemistry, root density, and BCS (first PCoA axis, henceforth bacterial PCo1) on the other hand, we used multiple linear regressions. Soil chemistry variables showed multi-collinearity, therefore we used principal component analysis to summarize variability in soil chemistry, with all input variables scaled and centered. The first principal component (soil PC1) was mostly driven by positive OMC and soil moisture (SI Fig. S2) and was used as a proxy for soil chemistry in the multiple regressions. Regression models were fit separately for bulk and intrinsic potential respiration, using soil PC1 only, or adding bacterial PCo1 and/or root density (scaled and centered) as predictors. To investigate how predictors of potential respiration differed between control and deep-thaw soils, we included treatment and its interactions with the other independent predictors in all models. We further fitted models with bacterial PCo1 alone to compare the relative importance of soil PC1 and bacterial PCo1. For both bulk and 
Table 1 Effects of decadal in situ deep-thaw and depth on soil abiotic variables, respiration rates, and bacterial community structure (BCS); association between environmental variables and $\mathrm{BCS}$

\begin{tabular}{|c|c|c|c|c|c|c|}
\hline \multirow[t]{2}{*}{ Soil chemistry and respiration (ANOVA) } & \multicolumn{2}{|c|}{$\begin{array}{l}\text { Decadal deep thaw } \\
\text { (treatment) }\end{array}$} & \multicolumn{2}{|l|}{ Depth } & \multicolumn{2}{|c|}{$\begin{array}{l}\text { Treatment: } \\
\text { depth }\end{array}$} \\
\hline & $F$ & $p$ & $F$ & $p$ & $F$ & $p$ \\
\hline Square-root organic matter content (OMC) & 2.839 & 0.123 & 24.972 & $<10^{-4}$ & 0.320 & 0.730 \\
\hline $\mathrm{pH}$ & 1.365 & 0.270 & 4.847 & 0.019 & 6.797 & 0.006 \\
\hline Log ammonium & 0.074 & 0.792 & 0.936 & 0.409 & 3.260 & 0.060 \\
\hline Moisture (gravimetric) & 2.012 & 0.187 & 7.795 & 0.003 & 1.126 & 0.344 \\
\hline Log bulk respiration (per g dry weight) & 5.254 & 0.045 & 19.764 & $<\mathbf{1 0}^{-4}$ & 0.658 & 0.529 \\
\hline Log intrinsic respiration (per g OM) & 0.232 & 0.641 & 0.417 & 0.665 & 3.920 & $\mathbf{0 . 0 3 7}$ \\
\hline$Q_{10}\left(11-21^{\circ} \mathrm{C}\right)$ & 3.360 & 0.097 & 5.770 & 0.011 & 4.101 & $\mathbf{0 . 0 3 2}$ \\
\hline Bacterial community structure (BCS) & Dev & $p$ & Dev & $p$ & Dev & $p$ \\
\hline ManyGLM & 1579 & 0.006 & 3711 & 0.001 & 1101 & 0.001 \\
\hline Association with BCS (envfit) & PCo1 & $\mathrm{PCo} 2$ & $R^{2}$ & $p$ & & \\
\hline Organic matter content (OMC) & -0.990 & -0.144 & 0.231 & 0.014 & & \\
\hline $\mathrm{pH}$ & 0.819 & 0.573 & 0.111 & 0.147 & & \\
\hline Ammonium & -0.113 & 0.994 & 0.317 & 0.004 & & \\
\hline Moisture (gravimetric) & -1.000 & 0.005 & 0.234 & 0.015 & & \\
\hline Root density & -0.804 & -0.595 & 0.233 & 0.022 & & \\
\hline
\end{tabular}

Bold text denotes significant $p$-values $(p<0.05)$ intrinsic potential respiration, we compared regression models based on their second-order Akaike's Information Criterion (AICc, AICcmodavg package [79]).

\section{Results}

\section{Soil chemistry and root density}

Soil moisture, organic matter, and carbon content were unaffected by the deep-thaw manipulation and were lower in the permafrost layer relative to the other two soil layers for both treatments. $\mathrm{pH}$ was higher in the permafrost than in the active layer in control soils but not in deep-thaw, while $\mathrm{NH}_{4}{ }^{+}$was unaffected by depth and treatment (Table 1, SI Table S3, SI Fig. S3). Roots cannot grow into frozen soil, therefore root density distribution reflected whether the soil was seasonally thawed or not (SI Fig. S4). Most of the root biomass belonged to sedges and shrubs.

\section{Bacterial community structure}

BCS varied strongly with depth and was also affected by the decadal in situ deep-thaw manipulation, but this effect differed between soil layers (Table 1, Fig. 1). Overall, BCS did not differ across the three seasonally thawed soil layers (active layer in both treatments, intermediate layer in deepthaw treatment; Fig. 1a). The three permanently frozen soil layers (intermediate layer in control treatment and permafrost layer in both treatments) differed from the seasonally thawed cluster (Fig. 1a, SI Table S4). Seasonal thawing in the intermediate layer thus led to a BCS undistinguishable from the active layer within a decade. This shift from perennially frozen to seasonally thawed BCS was characterized at the phylum level by large decreases in Firmicutes and Caldiserica, and an increase in Acidobacteria relative abundances (Fig. 1b). Soil moisture content, OMC, and root density were mainly associated with PCo1, reflecting the strong effects of depth and thawing, while $\mathrm{NH}_{4}{ }^{+}$was associated with $\mathrm{PCo}$.

Although in the active layer the BCS did not significantly differ between treatments, 321 OTUs showed significant changes (SI Fig S5). Eight relatively abundant OTUs ( $>0.5 \%$ of the rarefied reads) were more abundant in deep-thaw plots, of which four were putative aerobes or micro-aerobes and two were putative anaerobes (SI Table S2).

In the intermediate layer, 149 OTUs changed relative abundance with deep thaw, of which 9 were particularly abundant (Fig. 1c). Among these, the relative abundance of 6 obligate and putative anaerobes decreased, while putative aerobes had an increased relative abundance under deep thaw (SI Table S2).

In the permafrost layer, 48 OTUs changed relative abundance with deep thaw (SI Fig S5), even though no phase change was observed, of which 1 unclassified OTU was abundant and its relative abundance increased in the deep-thaw treatment (SI Table S2). 

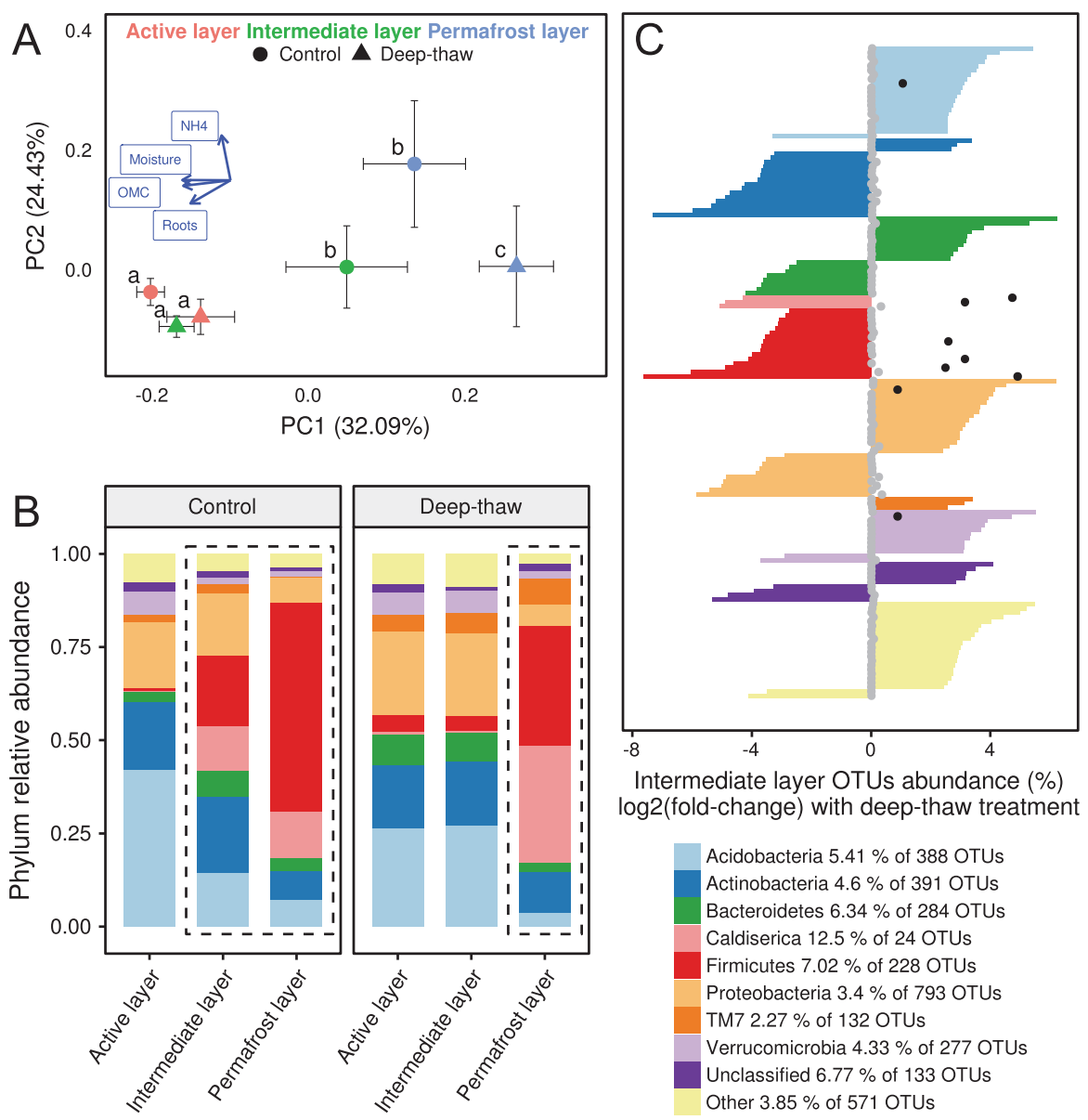

Actinobacteria $4.6 \%$ of 391 OTUs
Bacteroidetes $6.34 \%$ of 284 OTUs
Caldiserica $12.5 \%$ of 24 OTUs
Firmicutes $7.02 \%$ of 228 OTUs
Proteobacteria $3.4 \%$ of 793 OTUs
TM7 $2.27 \%$ of 132 OTUs
Verrucomicrobia $4.33 \%$ of 277 OTUs
Unclassified $6.77 \%$ of 133 OTUs
Other $3.85 \%$ of 571 OTUs

Fig. 1 Bacterial community structure based on 16S rRNA V3 region. a Principal coordinates analysis of weighted UniFrac distances; markers and error bars represent mean and standard error, respectively, of coordinates on the ordination axes for each depth $\times$ treatment combination $(n$ $=6$, except intermediate layer-deep thaw where $n=5$ ); different letters denote significantly different communities (manyglm post hoc, see SI Table S4). b Mean relative abundance of phyla in each depth $\times$ treatment combination; dashed rectangles enclose permanently frozen samples. c

\section{Potential aerobic respiration}

Bulk potential respiration rates (per g soil dry weight) as measured in aerobic soil incubations were 85\% (55-95\% \pm SE, $P<$ $0.001)$ lower in the permafrost layer than in the overlying layers, and were further reduced by $61 \%(41-74 \%, P=0.045)$ in the deep-thaw treatment across all soil layers (no depth $\times$ treatment interaction; Table 1, Fig. 2a). In contrast there was no overall effect of depth or treatment on intrinsic potential respiration (per $\mathrm{g}$ organic matter dry weight), but a significant depth $\times$ treatment interaction $(P=0.037$, Table 1, Fig. 2b), reflecting lower potential respiration by $45 \%(26-59 \%)$ in the active, $18 \%$ $(-10-39 \%)$ in the intermediate but higher by $74 \%(30-134 \%)$ in the permafrost layer, in soils from the deep-thaw plots than from the control plots. Similarly, there was no main effect of
Phylum distribution, abundance, and abundance change of the 149 OTUs that are different in the intermediate layer between the deep-thaw and control treatments (DEseq2 NB Wald test). Each bar represents the $\log 2$ of the fold change between control and deep-thaw samples for one OTU; dots represent the relative abundance of the OTU in the entire rarefied dataset, black dots indicate abundant OTUs $(>0.5 \%$, SI Table S2); number of OTUs in the legend is for the intermediate layer, while the percentages show what portion of those is in (c)

treatment or depth on the temperature sensitivity of respiration $\left(Q_{10}\right.$, calculated between 11 and $21^{\circ} \mathrm{C}$ ) but a significant depth $\times$ treatment interaction indicating that $Q_{10}$ varied between the intermediate and permafrost layer primarily in the deep-thaw plots (Table 1, SI Fig. S6, SI Table S5).

\section{Abiotic and biotic drivers of potential respiration}

Including the experimental treatment alongside soil chemistry (soil PC1), BCS (bacterial PCo1), and/or root density improved all regression models for both bulk and intrinsic potential respiration (SI Table S6). Bulk respiration models best fit the data when adding treatment without interactions (treatment effect on intercept) while for intrinsic respiration all interactions between treatment and other predictors 

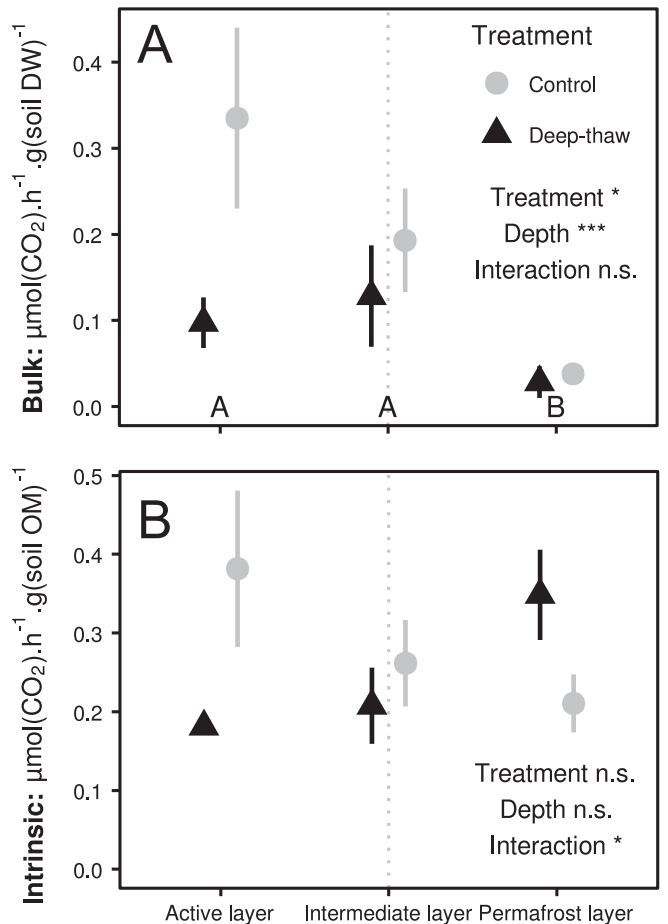

Fig. 2 Potential aerobic respiration of soils from different depths in control and decadal in situ deep-thaw plots during laboratory incubation at $11^{\circ} \mathrm{C}$, measured for 1 week after 1 month pre-incubation, expressed per gram soil dry weight (a) and per gram soil organic matter (b); samples to the right of the dotted line are from permanently frozen soil layers; means $\pm \operatorname{SE}(n=6)$. Asterisks denote significant effects $(* P<0.05 ; * * * P<0.001 ;$ n.s. non significant), letters denote significant differences between depths

significantly improved model fits (effect on slopes and intercept; Fig. 3, SI Table S6). Although soil PC1 slopes did not differ from zero, the significantly different slopes between control and deep-thaw samples for both bacterial PCo1 and soil PC1 reflected the depth $\times$ treatment interaction on intrinsic respiration previously described (Figs. 2b, 3, and Table 2).

Models combining BCS and soil chemistry best described both bulk and intrinsic potential respiration, with decreases in AICc compared to models based on soil chemistry only of 8.43 and 4.33, respectively (Table 2). When including bacterial PCo1 only, the fit for bulk respiration was worse, but for intrinsic respiration better than in models with soil $\mathrm{PC} 1$ alone $\left(\Delta \mathrm{AICc}-5.2, \Delta R^{2}+9 \%\right)$. The model using bacterial PCo1 only for intrinsic respiration was similarly well-fitting as the model including both soil PC1 and bacterial PCo1 $(\triangle \mathrm{AICc}$ $-0.88, \Delta R^{2}-8 \%$, likelihood-ratio test $P=0.059$, Table 2).

\section{Discussion}

In line with our hypothesis (a), the response of the BCS to the deep-thaw treatment was strongest in the intermediate

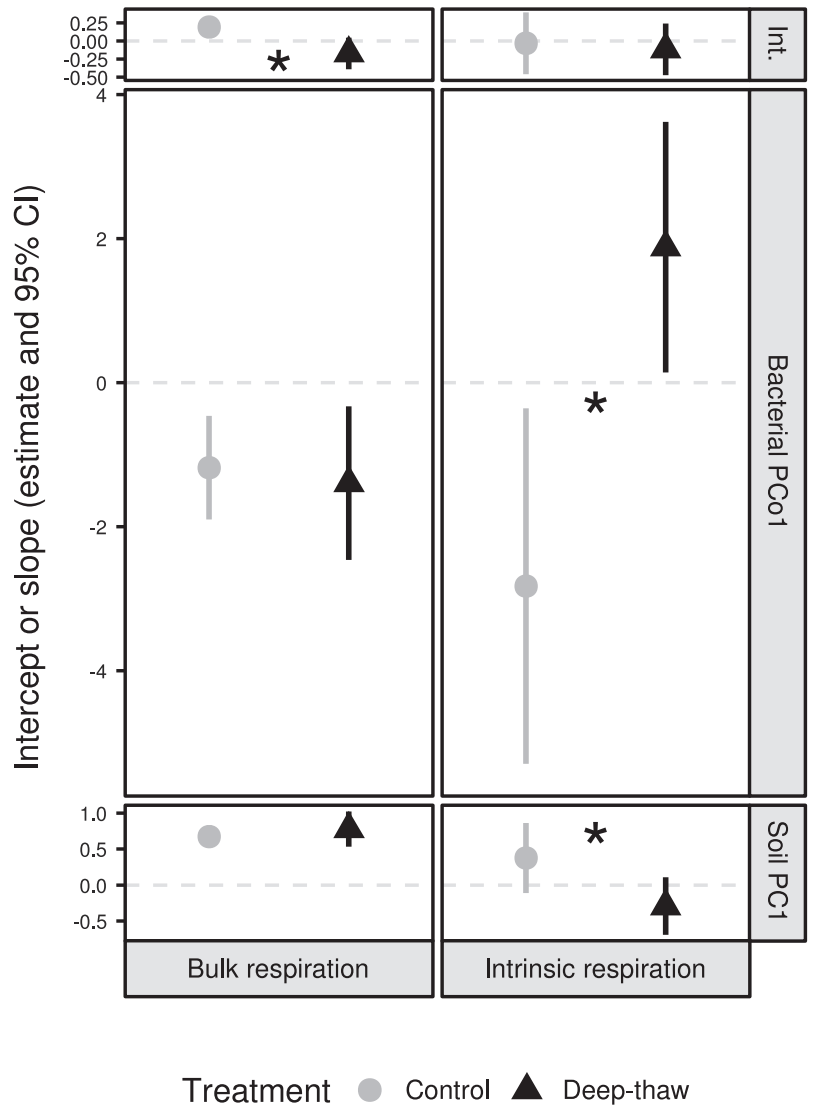

Fig. 3 Intercepts and slopes $(95 \% \mathrm{CI})$ of the best-fitting multiple linear regressions of respiration against soil chemistry (soil PC1) and bacterial community structure (bacterial PCo1). Asterisks denote significant differences between treatments $(P<0.05)$. Int. intercept

layer where the permafrost changed from permanently frozen to seasonally thawed (Fig. 1). The convergence of the deep-thaw intermediate layer BCS with the active layer communities supports previous findings of BCS differing between active layer and permafrost in the field [26, 81], and of convergence toward BCS found in the active layer upon permafrost thawing in vitro [28]. Furthermore, although the convergence might also partly stem from the differential growth of certain endogenous taxa, the strikingly similar communities among seasonally thawed soils suggests that overlying soil microorganisms migrated to newly thawed soil. One previous study has compared BCS at the same depth in seasonally thawed and permafrost soil in sites with permafrost degradation following fire, but there, fire and thaw effects were confounded [80]. Our results therefore provide the first in situ experimental evidence of the BCS response to permafrost thaw. Long-term freezing constraints can be a more important determinant of BCS than soil depth per se in permafrost soils, and once those constraints are relieved the overlying microorganisms seem to migrate, determining the resulting BCS. 


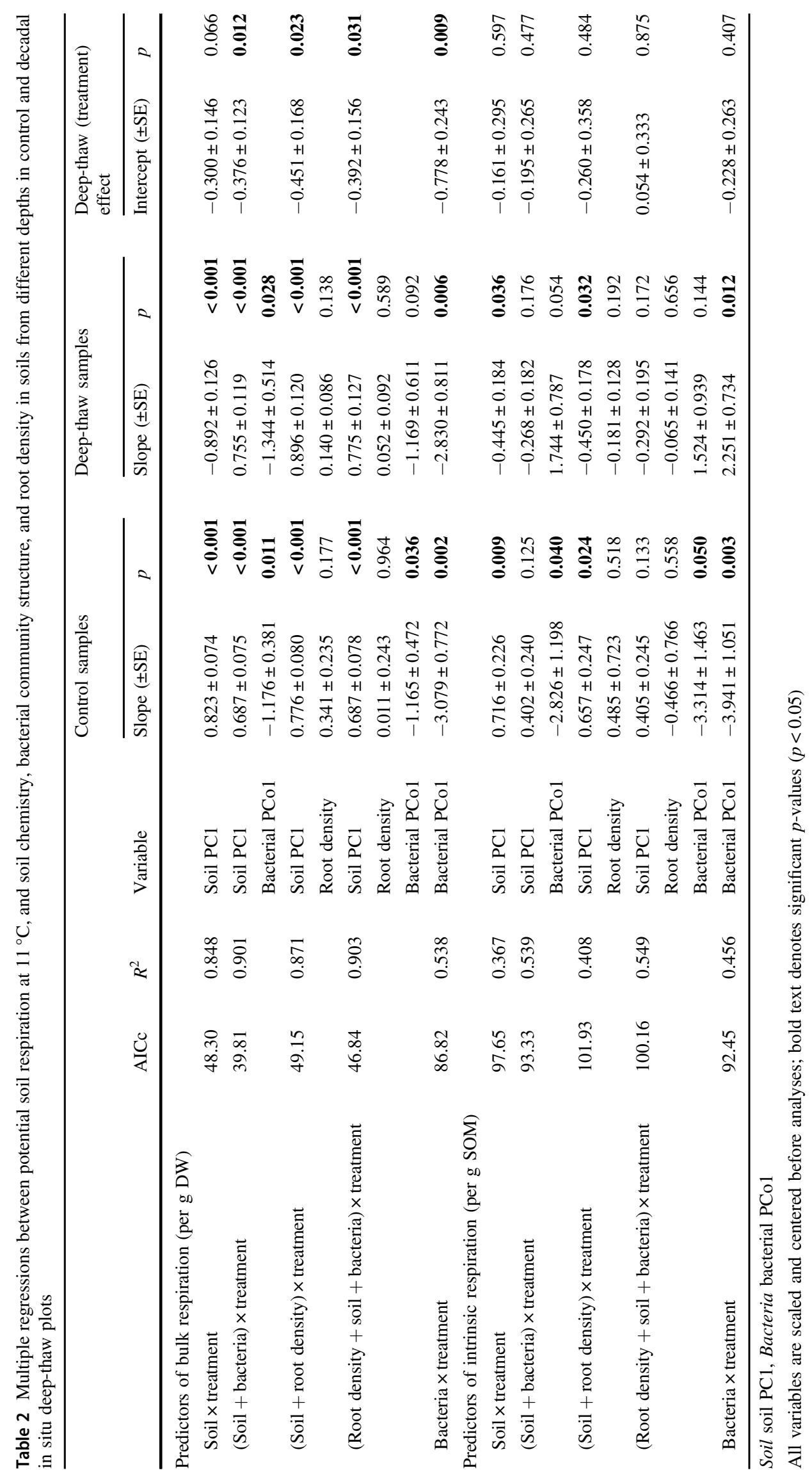


Although the deep-thaw experiment increased soil moisture in the upper soil layers [41], this effect did not extend to the deeper soil layers where we took our samples. Instead, increases in relative abundances of putative aerobic and anaerobic OTUs between treatments suggest that oxygen might have reached deeper soil layers in the deep-thaw plots, where E. vaginatum is more abundant. Eriophorum roots harbor aerenchymae, likely making deep soil more oxic (SI Fig. S4 \& S7 [13, 17, 81]).

High relative abundances (up to $60 \%$ ) of the phylum Caldiserica were found in the permanently frozen soil layers (Fig. 1b). This poorly known phylum has one cultured representative (thermophilic Caldisericum exile [82]), and has been found in other extreme environments (e.g. [83]). Wurzbacher et al. [84] recently showed that enigmatic microbial phyla dominate permafrost thaw ponds. The high relative abundances we observed suggest that intact permafrost, at least in our study system, could be used to investigate Caldiserica ecology, through culturing and -omics approaches.

As hypothesized (b), soils from the active layer in the deep-thaw plots had lower potential respiration rates than their control counterparts (Fig. 2). In contrast, higher ecosystem respiration in deep-thaw plots was observed in the first years of this experiment [40], when the increase in ALT, and thus in thawed SOM, was still limited $(\sim 6 \mathrm{~cm})$. We suggest that enhanced SOM decomposition and processing due to higher temperatures and prolonged thaw period over a decade of manipulation have increasingly depleted the labile C-pool, as Semenchuk et al. [9] suggested in a similar experiment.

In the intermediate layer, our hypothesis (b) of higher potential respiration because of increased microbial functional diversity was refuted: instead, both bulk and intrinsic potential respiration appeared lower in the deep-thaw plots. Upon permafrost thawing, fast metabolic responses facilitate SOM decomposition [28, 85], which could have increased carbon losses in the short term. Short periods of oxygenation, e.g., through E. vaginatum aerenchymae, can have lasting consequences on decomposition, by promoting phenol oxidase activity $[18,86]$, and seem plausible considering the observed changes in aerobic and anaerobic OTU relative abundances. Moreover, similar temperature effects as described above could result in the lower respiration rates observed after 10 years, through labile Cpool depletion, although we presumed the shorter thaw period in intermediate layers would delay such effects. This is further supported by higher $Q_{10}$ values in the deep-thaw intermediate layer, suggesting increased chemical recalcitrance (SI Fig. S6 [87]). While labile C-pool depletion has been previously suggested to explain decreased soil respiration $[9,88]$ and would fit our observations, integrated soil respiration measurements over a long-term experiment coupled with organic matter profiling would be necessary to confirm this interpretation.

Deep thaw had contrasting effects in the permafrost layer, with decreased bulk respiration and increased intrinsic respiration. Deep thaw may raise temperatures in stillfrozen soil layers at most by $<1{ }^{\circ} \mathrm{C}$ [74]. Combined with the observed higher rate of intrinsic respiration, it therefore seems unlikely that the aforementioned labile carbon depletion would also apply in the frozen soil. Alternatively, the different BCS in deep-thaw and control permafrost layers (Fig. 1a) might be functionally distinct, potentially leading to the observed differences in respiration. However, we hesitate to propose this as the single underlying mechanism, since the only abundant OTU affected by the manipulation provides no functional insights due to its high divergence from all cultured organisms (OTU_6022, SI Table S2). Further, although the treatment-affected OTUs may reflect a temperature effect on BCS in the permafrost layer, their relatively low number could also indicate spatial sampling effects exacerbated by the low population sizes in permafrost soils.

Our hypothesis (c), according to which including biota improves soil chemistry-based predictions of potential respiration, was supported for both bulk and intrinsic respiration: including BCS resulted in better-fitting models. Moreover, the significant interactions of soil chemistry and BCS with treatment, as well as the opposite directions of the slopes between treatments, strongly suggest that the drivers of intrinsic respiration fundamentally changed under decadal deep thaw (intrinsic respiration increased with, e.g., higher OMC in control soils but decreased in deep-thaw soils). Although all models including BCS showed a significantly better fit than soil-only models, bulk respiration rates were almost equally well explained by soil chemistry alone. In contrast, BCS alone explained intrinsic respiration rates better than soil chemistry, implying that after accounting for different OMC, BCS can explain variation in respiration better than soil chemistry. We cannot rule out that microbial biomass or other soil chemistry measures, e.g., recalcitrance of SOM $[89,90]$, may explain additional variation in respiration. Further, if BCS and respiration share these unmeasured variables as common drivers, some of the variation we currently attribute to BCS could in fact be due to other factors. 16S-based community profiles, as used here, give only limited information about functional potential. However, our results are in line with recent evidence suggesting that bacterial taxonomic composition can explain a unique portion of variation in respiration (agricultural temperate soil [91]), and partly determines soil carbon dynamics [92], particularly in organic soils where microbial activity is less constrained [94-95]. While the underlying mechanisms in terms of community functionality are unclear, our results suggest that measuring BCS 
may improve predictions of the permafrost carbon feedback.

In contrast with our hypothesis (c), including root density did not improve models of bulk or intrinsic potential respiration. Root density might affect soil respiration as measured in incubations through legacy effects of either rhizosphere priming, depleting the SOM pool, or alterations of BCS in the rhizosphere. The limited contribution of root density to our models is in agreement with relatively small (4\%) priming effects observed in peatlands [96], while the rhizosphere-BCS effects may already be accounted for by the overall changed BCS, thus explaining only some of the information that BCS contributes. In addition to such legacy effects possibly observed in incubations, higher root density might also affect soil respiration directly in situ, e.g., by stimulating rhizosphere microbial activity or by increasing SOM turnover without affecting the size of the SOM pool. Using different experimental designs, such as root exclosures, might allow to disentangle direct and legacy effects of root colonization effects from other consequences of thawing [97].

Our in situ approach confirmed the large changes in BCS after experimental permafrost thaw previously observed in incubations [28, 85], and further suggests that colonization by active layer bacteria might determine the fate of BCS in thawing permafrost. While root density appeared uninformative in predicting respiration, the strong linkages between BCS and potential respiration challenge the view that in soils BCS is unimportant for C-cycling, at least in our system, particularly after accounting for variations in SOM content. This BCS-C-cycling link, and underlying functional implications, should be investigated in other permafrost-affected environments, as it could imply that current predictions for SOM decomposition in thawing permafrost omit an important component. Altogether, our results show that permafrost thaw indirectly affects SOM decomposition through large effects on its drivers, such as BCS, which might prove important in understanding and predicting the permafrost carbon feedback.

The data supporting the findings of this study are available in figshare with the identifier https://doi.org/10.6084/ m9.figshare.5977471; the sequence data have been deposited in Sequence Read Archive with accession numbers SAMN07445364-SAMN07445398 and SAMN07445422; the entire bioinformatics and statistical analysis pipeline is available at https://bitbucket.org/smonteux/monteux_deep_ thaw/.

Acknowledgements We thank Tuukka Mäkiranta, Pia Bartels, and the Arctic Ecosystems students (2015) for help during field work; and the Abisko Scientific Research Station for practical support.

Funding: This study was funded by a Wallenberg Academy Fellowship (KAW 2012.0152), Swedish Research Council (Dnr 621-20115444), and Formas (Dnr 214-2011-788) grants all attributed to ED.
JTW was supported by a postdoctoral fellowship from the Flemish Science Foundation (FWO).

\section{Compliance with ethical standards}

Conflict of interest The authors declare that they have no conflict of interest.

Open Access This article is licensed under a Creative Commons Attribution 4.0 International License, which permits use, sharing, adaptation, distribution and reproduction in any medium or format, as long as you give appropriate credit to the original author(s) and the source, provide a link to the Creative Commons license, and indicate if changes were made. The images or other third party material in this article are included in the article's Creative Commons license, unless indicated otherwise in a credit line to the material. If material is not included in the article's Creative Commons license and your intended use is not permitted by statutory regulation or exceeds the permitted use, you will need to obtain permission directly from the copyright holder. To view a copy of this license, visit http://creativecommons. org/licenses/by/4.0/.

\section{References}

1. Hugelius G, Strauss J, Zubrzycki S, Harden JW, Schuur EAG, Ping C-L, et al. Estimated stocks of circumpolar permafrost carbon with quantified uncertainty ranges and identified data gaps. Biogeosciences. 2014;11:6573-93.

2. Schuur EaG, McGuire AD, Schaedel C, Grosse G, Harden JW, Hayes DJ, et al. Climate change and the permafrost carbon feedback. Nature. 2015;520:171-9.

3. Jassey VEJ, Lamentowicz M, Bragazza L, Hofsommer ML, Mills RTE, Buttler A, et al. Loss of testate amoeba functional diversity with increasing frost intensity across a continental gradient reduces microbial activity in peatlands. Eur $\mathbf{J}$ Protistol. 2016;55:190-202.

4. Margesin R, Miteva V. Diversity and ecology of psychrophilic microorganisms. Res Microbiol. 2011;162:346-61.

5. Knoblauch C, Beer C, Sosnin A, Wagner D, Pfeiffer E-M. Predicting long-term carbon mineralization and trace gas production from thawing permafrost of Northeast Siberia. Glob Change Biol. 2013;19:1160-72.

6. Davidson EA, Janssens IA, Luo Y. On the variability of respiration in terrestrial ecosystems: moving beyond Q10. Glob Change Biol. 2006;12:154-64.

7. Dorrepaal E, Toet S, van Logtestijn RSP, Swart E, van de Weg MJ, Callaghan TV, et al. Carbon respiration from subsurface peat accelerated by climate warming in the subarctic. Nature. 2009;460:616-9.

8. Hicks Pries CE, van Logtestijn RSP, Schuur EAG, Natali SM, Cornelissen JHC, Aerts R, et al. Decadal warming causes a consistent and persistent shift from heterotrophic to autotrophic respiration in contrasting permafrost ecosystems. Glob Change Biol. 2015;21:4508-19.

9. Semenchuk PR, Christiansen CT, Grogan P, Elberling B, Cooper EJ. Long-term experimentally deepened snow decreases growingseason respiration in a low- and high-arctic tundra ecosystem. J Geophys Res Biogeosci. 2016;121:1236-48.

10. Keuper F, Bodegom PM, Dorrepaal E, Weedon JT, Hal J, Logtestijn RSP, et al. A frozen feast: thawing permafrost increases plant-available nitrogen in subarctic peatlands. Glob Change Biol. 2012;18:1998-2007.

11. Weedon JT, Aerts R, Kowalchuk GA, van Logtestijn R, Andringa $\mathrm{D}$, van Bodegom PM. Temperature sensitivity of peatland $\mathrm{C}$ and 
N cycling: does substrate supply play a role? Soil Biol Biochem. 2013;61:109-20.

12. Keuper F, Dorrepaal E, van Bodegom PM, van Logtestijn R, Venhuizen G, van Hal J, et al. Experimentally increased nutrient availability at the permafrost thaw front selectively enhances biomass production of deep-rooting subarctic peatland species. Glob Change Biol. 2017;23:4257-4266. https://doi.org/10.1111/ gcb.13804

13. Blume-Werry G. The hidden life of plants: fine root dynamics in northern ecosystems. Doctoral thesis, Umeå University; 2016. http://umu.diva-portal.org/smash/record.jsf?pid=diva2:954761.

14. Fontaine S, Barot S, Barré P, Bdioui N, Mary B, Rumpel C. Stability of organic carbon in deep soil layers controlled by fresh carbon supply. Nature. 2007;450:277-80.

15. Wild B, Schnecker J, Alves RJE, Barsukov P, Barta J, Capek P, et al. Input of easily available organic $\mathrm{C}$ and $\mathrm{N}$ stimulates microbial decomposition of soil organic matter in arctic permafrost soil. Soil Biol Biochem. 2014;75:143-51.

16. Wild B, Gentsch N, Čapek P, Diáková K, Alves RJE, Bárta J, et al. Plant-derived compounds stimulate the decomposition of organic matter in arctic permafrost soils. Sci Rep. 2016;6:25607.

17. Armstrong W. Oxygen diffusion from the roots of some British bog plants. Nature. 1964;204:801-2.

18. Freeman C, Ostle N, Kang H. An enzymic 'latch' on a global carbon store. Nature. 2001;409:149-149.

19. Fierer N, Jackson RB. The diversity and biogeography of soil bacterial communities. Proc Natl Acad Sci USA. 2006;103:626-31.

20. Tkacz A, Cheema J, Chandra G, Grant A, Poole PS. Stability and succession of the rhizosphere microbiota depends upon plant type and soil composition. ISME J. 2015;9:2349-59.

21. Krab EJ, Lantman IMVS, Cornelissen JHC, Berg MP. How extreme is an extreme climatic event to a subarctic peatland springtail community? Soil Biol Biochem. 2013;59:16-24.

22. Schnecker J, Wild B, Takriti M, Eloy Alves RJ, Gentsch N, Gittel A, et al. Microbial community composition shapes enzyme patterns in topsoil and subsoil horizons along a latitudinal transect in Western Siberia. Soil Biol Biochem. 2015;83:106-15.

23. Bier RL, Bernhardt ES, Boot CM, Graham EB, Hall EK, Lennon JT, et al. Linking microbial community structure and microbial processes: an empirical and conceptual overview. FEMS Microbiol Ecol. 2015;91:fiv113.

24. Nannipieri P, Ascher J, Ceccherini MT, Landi L, Pietramellara G, Renella G. Microbial diversity and soil functions. Eur J Soil Sci. 2003;68:12-26.

25. Juarez S, Nunan N, Duday A-C, Pouteau V, Chenu C. Soil carbon mineralisation responses to alterations of microbial diversity and soil structure. Biol Fertil Soils. 2013;49:939-48.

26. Hultman J, Waldrop MP, Mackelprang R, David MM, McFarland $\mathrm{J}$, Blazewicz SJ, et al. Multi-omics of permafrost, active layer and thermokarst bog soil microbiomes. Nature. 2015;521:208-12.

27. Mackelprang R, Saleska SR, Jacobsen CS, Jansson JK, Taş N. Permafrost meta-omics and climate change. Annu Rev Earth Planet Sci. 2016;44:439-62.

28. Mackelprang R, Waldrop MP, DeAngelis KM, David MM, Chavarria KL, Blazewicz SJ, et al. Metagenomic analysis of a permafrost microbial community reveals a rapid response to thaw. Nature. 2011;480:368-71.

29. Yergeau E, Hogues H, Whyte LG, Greer CW. The functional potential of high Arctic permafrost revealed by metagenomic sequencing, qPCR and microarray analyses. ISME J. 2010;4:1206-14.

30. Myers B, Webster KL, Mclaughlin JW, Basiliko N. Microbial activity across a boreal peatland nutrient gradient: the role of fungi and bacteria. Wetl Ecol Manag. 2012;20:77-88.
31. Bragazza L, Bardgett RD, Mitchell EAD, Buttler A. Linking soil microbial communities to vascular plant abundance along a climate gradient. New Phytol. 2015;205:1175-82.

32. Iversen CM, Sloan VL, Sullivan PF, Euskirchen ES, McGuire $\mathrm{AD}$, Norby RJ, et al. The unseen iceberg: plant roots in arctic tundra. New Phytol. 2015;205:34-58.

33. Myers-Smith IH, Elmendorf SC, Beck PSA, Wilmking M, Hallinger $\mathrm{M}, \mathrm{Blok} \mathrm{D}$, et al. Climate sensitivity of shrub growth across the tundra biome. Nat Clim Change. 2015;5:887-91.

34. Nguyen C. Rhizodeposition of organic $\mathrm{C}$ by plants: mechanisms and controls. Agronomie. 2003;23:375-96.

35. Steven B, Niederberger TD, Whyte LG. Bacterial and archaeal diversity in permafrost. In: Margesin PDR, editor. Soil biology. Permafrost soils. Berlin Heidelberg: Springer; 2009. p. 59-72.

36. Eilers KG, Debenport S, Anderson S, Fierer N. Digging deeper to find unique microbial communities: the strong effect of depth on the structure of bacterial and archaeal communities in soil. Soil Biol Biochem. 2012;50:58-65.

37. Murton JB. Global warming and thermokarst. In: Margesin PDR, editor. Soil biology. Permafrost soils. Berlin Heidelberg: Springer; 2009. p. 185-203.

38. Arctic climate impact assessment. Impacts of a warming Arctic: Arctic Climate Impacts Assessment. Cambridge University Press: Cambridge, UK. 2005. p. 1042, ISBN: 0521617782.

39. Natali SM, Schuur EAG, Webb EE, Pries CEH, Crummer KG. Permafrost degradation stimulates carbon loss from experimentally warmed tundra. Ecology. 2014;95:602-8.

40. Njuabe HM. Subarctic peatlands in a changing climate: greenhouse gas response to experimentally increased snow cover. MSc thesis. Lund University; 2011. http://lup.lub.lu.se/student-papers/ record/1971208

41. Johansson M, Callaghan TV, Bosio J, Akerman HJ, JackowiczKorczynski M, Christensen TR. Rapid responses of permafrost and vegetation to experimentally increased snow cover in subarctic Sweden. Environ Res Lett. 2013;8:35025.

42. Luczak C, Janquin M-A, Kupka A. Simple standard procedure for the routine determination of organic matter in marine sediment. Hydrobiologia. 1997;345:87-94.

43. Kirschbaum MUF. The temperature dependence of soil organic matter decomposition, and the effect of global warming on soil organic C storage. Soil Biol Biochem. 1995;27:753-60.

44. Bartram AK, Lynch MDJ, Stearns JC, Moreno-Hagelsieb G, Neufeld JD. Generation of multimillion-sequence 16S rRNA gene libraries from complex microbial communities by assembling paired-end Illumina reads. Appl Environ Microbiol. 2011;77:3846-52.

45. Rognes T, Flouri T, Nichols B, Quince C, Mahé F. VSEARCH: a versatile open source tool for metagenomics. PeerJ. 2016;4:e2584.

46. Edgar RC, Haas BJ, Clemente JC, Quince C, Knight R. UCHIME improves sensitivity and speed of chimera detection. Bioinformatics. 2011;27:2194-200.

47. Haas BJ, Gevers D, Earl AM, Feldgarden M, Ward DV, Giannoukos $\mathrm{G}$, et al. Chimeric 16S rRNA sequence formation and detection in Sanger and 454-pyrosequenced PCR amplicons. Genome Res. 2011;21:494-504.

48. Mackelprang R, Burkert A, Haw M, Mahendrarajah T, Conaway $\mathrm{CH}$, Douglas TA. Microbial survival strategies in ancient permafrost: insights from metagenomics. ISME J. 2017;11:2305-18.

49. Caporaso JG, Kuczynski J, Stombaugh J, Bittinger K, Bushman FD, Costello EK, et al. QIIME allows analysis of high-throughput community sequencing data. Nat Methods. 2010;7:335-6.

50. McDonald D, Price MN, Goodrich J, Nawrocki EP, DeSantis TZ, Probst A, et al. An improved Greengenes taxonomy with explicit ranks for ecological and evolutionary analyses of bacteria and archaea. ISME J. 2012;6:610-8. 
51. Price MN, Dehal PS, Arkin AP. FastTree 2-approximately maximum-likelihood trees for large alignments. PLoS ONE. 2010;5:e9490.

52. Wang Q, Garrity GM, Tiedje JM, Cole JR. Naive Bayesian classifier for rapid assignment of rRNA sequences into the new bacterial taxonomy. Appl Environ Microbiol. 2007;73:5261-7.

53. Lagkouvardos I, Fischer S, Kumar N, Clavel T. Rhea: a transparent and modular $\mathrm{R}$ pipeline for microbial profiling based on 16S rRNA gene amplicons. PeerJ. 2017;5:e2836

54. Lozupone C, Lladser ME, Knights D, Stombaugh J, Knight R. UniFrac: an effective distance metric for microbial community comparison. ISME J. 2011;5:169-72.

55. Love MI, Huber W, Anders S. Moderated estimation of fold change and dispersion for RNA-seq data with DESeq2. Genome Biol. 2014;15:550.

56. McMurdie PJ, Holmes S. Waste not, want not: why rarefying microbiome data is inadmissible. PLoS Comput Biol. 2014;10: e1003531.

57. Altschul SF, Gish W, Miller W, Myers EW, Lipman DJ. Basic local alignment search tool. J Mol Biol. 1990;215:403-10.

58. Emerson D, Field EK, Chertkov O, Davenport KW, Goodwin L, Munk C, et al. Comparative genomics of freshwater Fe-oxidizing bacteria: implications for physiology, ecology, and systematics. Front Microbiol. 2013;4:254.

59. Head IM, Hiorns WD, Embley TM, McCarthy AJ, Saunders JR. The phylogeny of autotrophic ammonia-oxidizing bacteria as determined by analysis of 16S ribosomal RNA gene sequences. Microbiology. 1993;139:1147-53.

60. Kim S, Jeong H, Chun J. Clostridium aestuarii sp. nov., from tidal flat sediment. Int J Syst Evol Microbiol. 2007;57:1315-7.

61. Liou JS-C, Balkwill DL, Drake GR, Tanner RS. Clostridium carboxidivorans sp. nov., a solvent-producing clostridium isolated from an agricultural settling lagoon, and reclassification of the acetogen Clostridium scatologenes strain SL1 as Clostridium drakei sp. nov. Int J Syst Evol Microbiol. 2005;55:2085-91.

62. Maszenan AM, Seviour RJ, Patel BK, Schumann P, Burghardt J, Tokiwa $\mathrm{Y}$, et al. Three isolates of novel polyphosphateaccumulating gram-positive cocci, obtained from activated sludge, belong to a new genus, Tetrasphaera gen. nov., and description of two new species, Tetrasphaera japonica sp. nov. and Tetrasphaera australiensis sp. nov. Int J Syst Evol Microbiol. 2000;50:593-603.

63. Rainey FA. Ruminococcaceae fam. nov. In: Bergey's manual of systematics of archaea and bacteria. Wiley; 2015. https://doi.org/ 10.1002/9781118960608.fbm00136.

64. Rainey FA, Hollen BJ, Small AM. Clostridium. In: W. B. Whitman, F. Rainey, P. Kämpfer, M. Trujillo, J. Chun, P. DeVos, B. Hedlund and S. Dedysh. Bergey's manual of systematics of archaea and bacteria. John Wiley \& Sons, Inc. 2015. https://doi. org/10.1002/9781118960608.gbm00619.

65. Spring S, Merkhoffer B, Weiss N, Kroppenstedt RM, Hippe H, Stackebrandt E. Characterization of novel psychrophilic clostridia from an Antarctic microbial mat: description of Clostridium frigoris sp. nov., Clostridium lacusfryxellense sp. nov., Clostridium bowmanii sp. nov. and Clostridium psychrophilum sp. nov. and reclassification of Clostridium laramiense as Clostridium estertheticum subsp. laramiense subsp. nov. Int J Syst Evol Microbiol. 2003;53:1019-29.

66. Tamura T, Ishida Y, Otoguro M, Yamamura H, Hayakawa M, Suzuki K-I. Angustibacter luteus gen. nov., sp. nov., isolated from subarctic forest soil. Int J Syst Evol Microbiol. 2010;60:2441-5.

67. Tanner RS, Miller LM, Yang D. Clostridium ljungdahlii sp. nov., an acetogenic species in clostridial rRNA homology group I. Int J Syst Evol Microbiol. 1993;43:232-6.

68. Ward NL, Challacombe JF, Janssen PH, Henrissat B, Coutinho $\mathrm{PM}, \mathrm{Wu} \mathrm{M}$, et al. Three genomes from the phylum Acidobacteria provide insight into the lifestyles of these microorganisms in soils. Appl Environ Microbiol. 2009;75:2046-56.

69. Wiegel J. Clostridiaceae. In: Bergey's manual of systematics of archaea and bacteria. Wiley; 2015. https://doi.org/10.1002/ 9781118960608.fbm00129

70. Xing D, Ren N, Li Q, Lin M, Wang A, Zhao L. Ethanoligenens harbinense gen. nov., sp. nov., isolated from molasses wastewater. Int J Syst Evol Microbiol. 2006;56:755-60.

71. Yoon J-H, Kang S-J, Oh T-K. Dokdonella koreensis gen. nov., sp. nov., isolated from soil. Int $\mathrm{J}$ Syst Evol Microbiol. 2006;56:145-50.

72. Zhang J-Y, Liu X-Y, Liu S-J. Phycicoccus cremeus sp. nov., isolated from forest soil, and emended description of the genus Phycicoccus. Int J Syst Evol Microbiol. 2011;61:71-5.

73. Basiliko N, Stewart H, Roulet NT, Moore TR. Do root exudates enhance peat decomposition? Geomicrobiol J. 2012;29:374-8.

74. R Core Team. R: a language and environment for statistical computing. R Foundation for Statistical Computing. R version 3.4.1. 2017. https://www.R-project.org

75. Pinheiro J, Bates D, DebRoy S, Sarkar D, R Core Team. nlme: linear and nonlinear mixed effects models. R package version 3.1131. 2017. https://CRAN.R-project.org/package $=$ nlme

76. Lenth R. Least-Squares Means: the R package lsmeans. J Stat Softw. 2016;69:1-33.

77. Wang Y, Naumann U, Wright ST, Warton DI. mvabund-an R package for model-based analysis of multivariate abundance data. Methods Ecol Evol. 2012;3:471-4.

78. Warton DI, Wright ST, Wang Y. Distance-based multivariate analyses confound location and dispersion effects. Methods Ecol Evol. 2012;3:89-101.

79. Oksanen J, Blanchet FG, Friendly M, Kindt R, Legendre P, McGlinn D, et al. vegan: community ecology package. R-package version 2.4-3. 2017. https://cran.r-project.org/package=vegan

80. Mazerolle MJ. AICcmodavg: model selection and multimodel inference based on (Q)AIC(c). R package version 2.1-1. 2017. https://cran.r-project.org/package $=$ AICcmodavg

81. Taş N, Prestat E, McFarland JW, Wickland KP, Knight R, Berhe AA, et al. Impact of fire on active layer and permafrost microbial communities and metagenomes in an upland Alaskan boreal forest. ISME J. 2014;8:1904-19.

82. Ström L, Mastepanov M, Christensen TR. Species-specific effects of vascular plants on carbon turnover and methane emissions from wetlands. Biogeochemistry. 2005;75:65-82.

83. Mori K, Yamaguchi K, Sakiyama Y, Urabe T, Suzuki K. Caldisericum exile gen. nov., sp. nov., an anaerobic, thermophilic, filamentous bacterium of a novel bacterial phylum, Caldiserica phyl. nov., originally called the candidate phylum OP5, and description of Caldisericaceae fam. nov., Caldisericales ord. nov. and Caldisericia classis nov. Int J Syst Evol Microbiol. 2009;59:2894-8.

84. Perner M, Gonnella G, Kurtz S, LaRoche J. Handling temperature bursts reaching $464^{\circ} \mathrm{C}$ : different microbial strategies in the sisters peak hydrothermal chimney. Appl Environ Microbiol. 2014;80:4585-98.

85. Wurzbacher C, Nilsson RH, Rautio M, Peura S. Poorly known microbial taxa dominate the microbiome of permafrost thaw ponds. ISME J. 2017;11:1938-41.

86. Coolen MJL, Orsi WD. The transcriptional response of microbial communities in thawing Alaskan permafrost soils. Terr Microbiol. 2015;6:197.

87. Brouns K, Verhoeven JTA, Hefting MM. Short period of oxygenation releases latch on peat decomposition. Sci Total Environ. 2014;481:61-8.

88. Conant RT, Drijber RA, Haddix ML, Parton WJ, Paul EA, Plante AF, et al. Sensitivity of organic matter decomposition to warming varies with its quality. Glob Change Biol. 2008;14:868-77. 
89. Christiansen CT, Svendsen SH, Schmidt NM, Michelsen A. High arctic heath soil respiration and biogeochemical dynamics during summer and autumn freeze-in-effects of long-term enhanced water and nutrient supply. Glob Change Biol. 2012;18:3224-36.

90. Johansson M, Akerman J, Keuper F, Christensen TR, Lantuit H, Callaghan TV. Past and present permafrost temperatures in the Abisko area: redrilling of boreholes. Ambio. 2011;40:558-65.

91. Dieleman CM, Lindo Z, McLaughlin JW, Craig AE, Branfireun BA. Climate change effects on peatland decomposition and porewater dissolved organic carbon biogeochemistry. Biogeochemistry. 2016;128:385-96.

92. Drake TW, Wickland KP, Spencer RGM, McKnight DM, Striegl RG. Ancient low-molecular-weight organic acids in permafrost fuel rapid carbon dioxide production upon thaw. Proc Natl Acad Sci USA. 2015;112:13946-51.
93. Liu Y-R, Delgado-Baquerizo M, Wang J-T, Hu H-W, Yang Z, He $\mathrm{J}-\mathrm{Z}$. New insights into the role of microbial community composition in driving soil respiration rates. Soil Biol Biochem. 2018;118:35-41.

94. Mau RL, Liu CM, Aziz M, Schwartz E, Dijkstra P, Marks JC, et al. Linking soil bacterial biodiversity and soil carbon stability. ISME J. 2015;9:1477-80.

95. Nunan N, Leloup J, Ruamps LS, Pouteau V, Chenu C. Effects of habitat constraints on soil microbial community function. Sci Rep. 2017;7:4280.

96. Schimel J, Schaeffer SM. Microbial control over carbon cycling in soil. Terr Microbiol. 2012;3:348.

97. Strickland MS, Lauber C, Fierer N, Bradford MA. Testing the functional significance of microbial community composition. Ecology. 2009;90:441-51. 